\title{
Ecological recipes for selecting community function
}

Simulations of eco-evolutionary processes involved in artificial selection of microbial communities provide a guide to optimize experimental design for improving ecosystem functions.

\section{Silvia De Monte}

Institut de Biologie de l'Ecole Normale Supérieure, Département de Biologie, Ecole Normale Supérieure, CNRS, INSERM, PSL Research University, Paris, France Department of Evolutionary Theory, Max Planck Institute for Evolutionary Biology, Plön, Germany silvia.de.monte@bio.ens.psl.eu

Microbial communities are increasingly seen as generalized forms of organisms $s^{1,2}$. In analogy ${ }^{3}$, their function (phenotype) results from the interaction of a multitude of 'species' (genes), whose abundances (levels of expression) feed back on themselves. This consideration of an overall community phenotype has led to the idea that selection can be applied at the community level to optimize functions, for example biomass production or the ability to consume or produce specific molecules. Community selection is possible when the community is spatially compartmentalized and a community's genealogy established ${ }^{4}$. However, it is unclear to what extent different options for experimental layout affect the capacity of those communities to respond to selection.

Writing in Nature Ecology \& Evolution, Chang et al. ${ }^{5}$ simulate different experimental designs for community selection in a common modelling framework. This stands as a tool to customize selection strategies for specific communities, as well as to formulate hypotheses on general principles underpinning community evolution. Through their new package ecoprospector, they simulate the dynamics of communities on two time-scales, within and across community generations (Fig. 1). Species interactions are mediated by resource consumption through a fixed set of parameters. In principle, and as increasingly realized in quantitative studies of microbial communities ${ }^{6-9}$, these can be measured independently of the selection experiment itself.

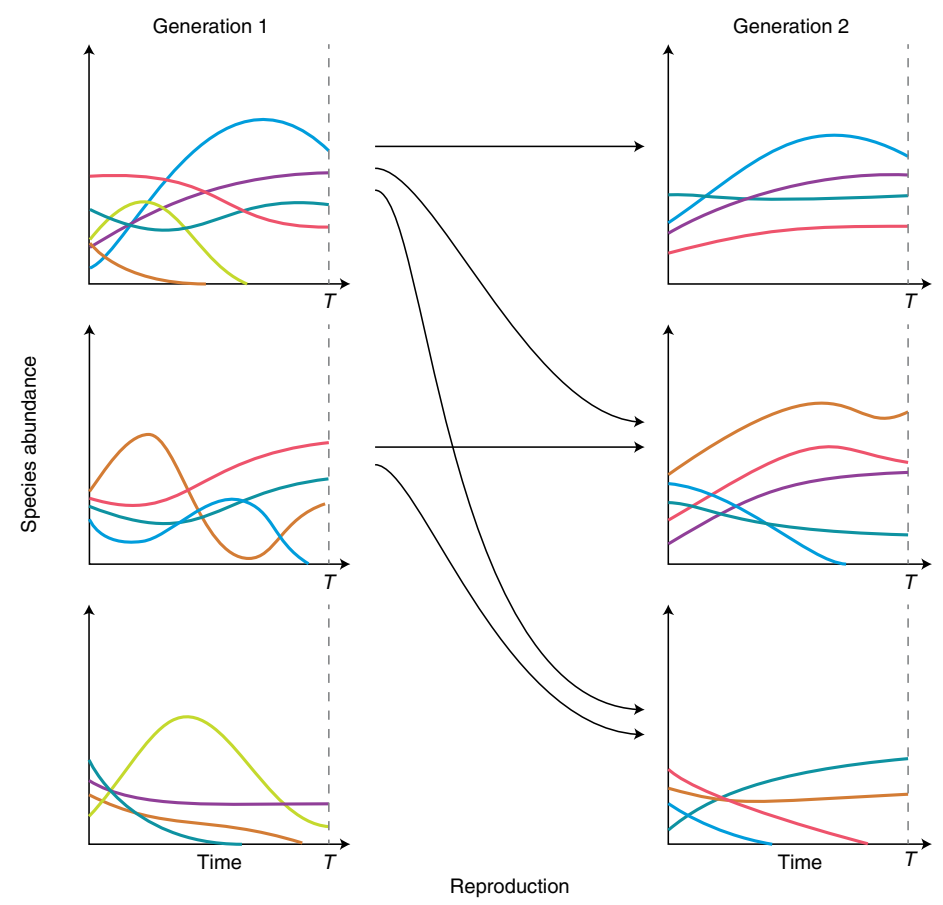

Figure 1: Schematic representation of ecology in an experiments of microbial community selection. Three initial communities are depicted, each starting with a different species composition and abundance (indicated by different colours). At each generation, species abundances undergo deterministic dynamics, reaching the 'adult' stage (at time T), when selection is applied on a community function (for example, on total biomass). At reproduction, sampling and dispersal from surviving communities set the initial state of the following community generation. Ecology drives changes of community-level traits on both time scales (within and between generations), even in the absence of evolution in the species traits. 
Chang and colleagues show that even if evolution does not act on species traits, ecology has farreaching consequences on the success of selection. The issue at the core of their numerical exploration is how variation in collective function is generated and maintained in a metapopulation of communities, and how it affects the efficacy of selection.

The authors note first that population dynamics are themselves a source of variation. This variation, however, is ephemeral (over generations, abundances tend to stabilize) and may sidetrack evolutionary progress by temporarily boosting communities that eventually manifest lower performance. They suggest that better and more reliable solutions are found when communities are allowed to reach a 'generationally stable' state - where, drawing upon the analogy to organisms, they undergo a repeatable 'development' - before selection is applied. This implies that the timing of selection should be adjusted to that of the ecology of each community.

A side effect of allowing communities to reliably self-organize over and again is, however, a reduction in the pool of functional variants from which selection can draw. Evolution of function across multiple generations would quickly grind to a halt, unless compositional variation is replenished. Multicellular organisms solved this problem by sexual reproduction, which shuffles alleles from a population gene pool. Artificially bred communities are in this respect excitingly flexible. Chang and colleagues test different rules for producing offspring communities from a set of parents. First, they show that - as for the 'stochastic corrector' mode ${ }^{10}$ - sampling (for example, by dilution) can improve community function. Second, they consider mixing of parental communities as a means to generate compositional novelty, which allows exploration of the combinatorially large space of functions that a given pool of species can attain. They conclude that combining these two recipes for enhancing variation in species composition and abundance is the most efficient way to select community function.

Unfortunately, even in the best scenarios identified by these authors, the increase in community function by purely ecological means appears to be quantitatively modest.

Indeed, functional improvement is bounded by the number of generationally stable solutions available to selection, which could be quite small for communities of a few tens of species. This limitation could be overcome if species-level parameters evolved, as they easily do in microbial populations. This would complicate matters considerably. On the one hand, conflicts may emerge between communities and their composing species. On the other hand, encompassing intra-specific variation would drastically expand the already large parameter space, making it practically unfeasible to assess the generality of simulation results.

One way forward to identify over-arching principles for community evolution in these more general settings is to focus on consortia comprised of few species, with the expectation that results can be scaled up from simple to complex communities. Several recent studies ${ }^{11-14}$ suggest that, as selection acts at the community level, evolution reduces conflicts between species and enhances interactions.

In contrast, theoretical understanding is still scant for the case when communities are composed of many species. Simulations of evolving large ecosystems have found that only certain selection targets promoted species interactions ${ }^{15}$. Moreover, when this happened, dispersal at reproduction predominantly produced derived communities in which only a few species accounted for the selected function. Previously discussed recipes for boosting variation may thus become a hindrance when selecting for stable and diverse multi-species assemblies. Further studies are needed to evaluate if and when this is the case.

Additional insights may stem from viewing large communities through the lens of their global properties, just as one would look to organismal traits as products of gene networks - even without 
knowing detail of their regulation. Leveraging on statistical descriptions of large networks of interacting species ${ }^{16}$, one can hope to describe the system-level effects of selection and understand how evolutionary trajectories deploy. Such an approach, moreover, opens the door to the study of communities whose ecology is out-of-equilibrium ${ }^{17,18}$, where generational stability may be unattainable even in the simplest experimental designs. Ultimately, these theoretical studies might also bring novel perspectives on how organisms evolve.

\section{References:}

1. Doolittle, W. F. \& Zhaxybayeva, O. Metagenomics and the Units of Biological Organization. BioScience 60, 102-112 (2010).

2. Liautaud, K., van Nes, E. H., Barbier, M., Scheffer, M. \& Loreau, M. Superorganisms or loose collections of species? A unifying theory of community patterns along environmental gradients. Ecol. Lett. (2019).

3. Morris, A., Meyer, K. \& Bohannan, B. Linking microbial communities to ecosystem functions: what we can learn from genotype-phenotype mapping in organisms. Philos. Trans. R. Soc. B Biol. Sci. 375, 20190244 (2020).

4. De Monte, S. \& Rainey, P. B. Nascent multicellular life and the emergence of individuality. J. Biosci. (2014)

5. Chang et al. Top-down engineering of complex communities by directed evolution. Nature Ecology \& Evolution

6. Goldford, J. E. et al. Emergent simplicity in microbial community assembly. Science 361, 469-474 (2018).

7. Machado, D. et al. Polarization of microbial communities between competitive and cooperative metabolism. Nat. Ecol. Evol. 5, 195-203 (2021).

8. Ratzke, C., Barrere, J. \& Gore, J. Strength of species interactions determines biodiversity and stability in microbial communities. Nat. Ecol. Evol. 4, 376-383 (2020).

9. Wright, E. S. \& Vetsigian, K. H. Inhibitory interactions promote frequent bistability among competing bacteria. Nat. Commun. 7, 11274 (2016).

10. Szathmáry, E. \& Demeter, L. Group selection of early replicators and the origin of life. J. Theor. Biol. 128, 463-486 (1987).

11. Xie, L., Yuan, A. E. \& Shou, W. Simulations reveal challenges to artificial community selection and possible strategies for success. PLOS Biol. 17, e3000295 (2019).

12. Vliet, S. van \& Doebeli, M. The role of multilevel selection in host microbiome evolution. Proc. Natl. Acad. Sci. 116, 20591-20597 (2019).

13. Doulcier, G., Lambert, A., De Monte, S. \& Rainey, P. B. Eco-evolutionary dynamics of nested Darwinian populations and the emergence of community-level heredity. elife 9, e53433 (2020).

14. Black, A. J., Bourrat, P. \& Rainey, P. B. Ecological scaffolding and the evolution of individuality. Nat. Ecol. Evol. 4, 426-436 (2020).

15. Williams, H. T. P. \& Lenton, T. M. Artificial selection of simulated microbial ecosystems. Proc. Natl. Acad. Sci. 104, 8918-8923 (2007).

16. Barbier, M., Arnoldi, J.-F., Bunin, G. \& Loreau, M. Generic assembly patterns in complex ecological communities. Proc. Natl. Acad. Sci. 115, 2156-2161 (2018).

17. Pearce, M. T., Agarwala, A. \& Fisher, D. S. Stabilization of extensive fine-scale diversity by ecologically driven spatiotemporal chaos. Proc. Natl. Acad. Sci. 201915313 (2020).

18. Roy, F., Barbier, M., Biroli, G. \& Bunin, G. Complex interactions can create persistent fluctuations in high-diversity ecosystems. PLOS Comput. Biol. 16, e1007827 (2020). 